\title{
Temperature sensing using an optical time domain reflectometer and mechanical long-period fiber gratings fabricated from a heat- shrinkable tube
}

\author{
Yasuhiro Tsutsumia), Masaharu Ohashi, and Yuji Miyoshi \\ Department of Electrical and Information Systems, Graduate school of engineering, \\ Osaka Prefecture University, 1-1 Gakuen-cho, Naka Sakai, Osaka 599-8531, Japan
}

a)TsutsumiYasuhiro@mem.iee.or.jp

Abstract: We develop a simple temperature sensing using an optical time domain reflectometer (OTDR) and a mechanical long-period fiber grating (LPFG) fabricated from a heat-shrinkable tube and a metric screw. The resonance loss of the fabricated LPFG is strongly dependent on temperature as temperature-dependent heat-shrinkage of the tube produces a change in the pressure applied to the fiber. In our proposed temperature sensor, the LPFG insertion loss due to a change in the pressure applied to the fiber is measured using an OTDR with a resonance wavelength. Both singlepoint and multi-point temperature sensing is successfully demonstrated experimentally.

Keywords: long period fiber gratings, optical time domain reflectometer, temperature sensing

Classification: Sensing

\section{References}

[1] S. Nam, C. Zhan, J. Lee, C. Hahn, K. Reichard, P. Ruffin, K.-L. Deng, and S. Yin, "Bend-insensitive ultra short long-period gratings by the electric arc method and their applications to harsh environment sensing and communication," Opt. Express, vol. 13, pp. 731-737, 2005. DOI:10.1364/OPEX.13. 000731

[2] H. Xuan, W. Jin, and M. Zhang, " $\mathrm{CO}_{2}$ laser induced long period gratings in optical microfibers,” Opt. Express, vol. 17, pp. 21882-21890, 2009. DOI:10. 1364/OE.17.021882

[3] S. W. James and R. P. Tatam, "Optical fibre long-period fiber grating sensors: characteristics and application," Meas. Sci. Technol., vol. 14, no. 5, pp. R49R61, 2003. DOI:10.1088/0957-0233/14/5/201

[4] V. Bhatia and A. M. Vengsarkar, "Optical fiber long-period fiber grating sensors," Opt. Lett., vol. 21, no. 9, pp. 692-694, 1996. DOI:10.1364/OL.21. 000692

[5] Y. Wang, "Review of long period fiber gratings written by CO2 laser," J. Appl. 
Phys., vol. 108, pp. 081101, 2010. DOI:10.1063/1.3493111

[6] L. Drozin, P. Y. Fonjallaz, and L. Stensland, "Long-period fiber gratings written by $\mathrm{CO} 2$ exposure of $\mathrm{H} 2$ loaded standard fibers," Electron. Lett., vol. 36, pp. 742-744, 2000. DOI:10.1049/el:20000510

[7] G. Rego, O. Okhotnikov, E. Dianov, and V. Sulimov, "High-temperature stability of long-period fibre gratings using an electric arc," J. Lightwave Technol., vol. 19, pp. 1574-1579, 2001. DOI:10.1109/50.956145

[8] P. Palai, M. N. Satyanarayan, M. Das, K. Thyagarajan, and B. P. Pal, "Characterization and simulation of long period gratings fabricated using electric discharge," Opt. Commun., vol. 193, pp. 181-185, 2001. DOI:10. 1016/S0030-4018(01)01231-7

[9] S. Savin, M. J. F. Digonnet, G. S. Kino, and H. J. Shaw, "Tunable mechanically induced long-period fiber gratings," Opt. Lett., vol. 25, pp. 710-712, 2000. DOI:10.1364/OL.25.000710

[10] H. Sakata and K. Yamashita, "Magnetic-force-induced long-period fiber gratings," Opt. Lett., vol. 37, pp. 1250-1252, 2012. DOI:10.1364/OL.37. 001250

[11] Y. Tsutsumi, M. Ohashi, and Y. Miyoshi, "Temperature-sensitive mechanical LPFG using contractive force of heat-shrinkable tube," Opt. Fiber Technol., vol. 19, pp. 55-59, 2013. DOI:10.1016/j.yofte.2012.11.002

[12] T. Erdogan, "Fiber grating spectra," J. Lightwave Technol., vol. 15, no. 8, pp. 1277-1294, 1997. DOI:10.1109/50.618322

\section{Introduction}

A long-period fiber grating (LPFG) is formed in a single-mode fiber, which enables light to be coupled from the fiber core to selected cladding modes at specific resonance wavelengths. LPFGs have been extensively investigated for use in optical communication and sensing applications because of their sensitivity to strain, temperature, and refractive index $[1,2,3]$.

Numerous techniques are available for fabricating LPFGs. Approaches that have been demonstrated include periodic local heating of the fiber using a $\mathrm{CO} / \mathrm{CO}_{2}$ laser $[4,5]$ or electric arc discharge $[6,7]$. Fabrication techniques for mechanically induced LPFGs have also been reported [8, 9, 10]. Mechanically induced LPFGs are attractive because of their simplicity, flexibility, and cost effectiveness. In a recent paper, we proposed a new mechanically induced LPFG fabricated from a heat-shrinkable tube and a metric screw and reported its temperature characteristics [11].

In the current study, we propose a simple temperature-sensing using an optical time domain reflectometer (OTDR) and an LPFG fabricated from a heat-shrinkable tube and a metric screw. In the proposed sensing, the OTDR senses the temperature from the LPFG insertion loss that occurs when pressure is applied to the fiber. We demonstrate successfully both single-point and multi-point temperature sensing.

\section{Temperature sensing using an OTDR and LPFG}

Fig. 1 shows the temperature measurement configuration. Temperature sensing is achieved by monitoring the insertion loss at a wavelength near the resonance wavelength of the LPFG. The transmitted spectrum changes significantly in line 
(a)

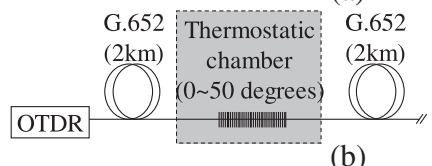

(b)

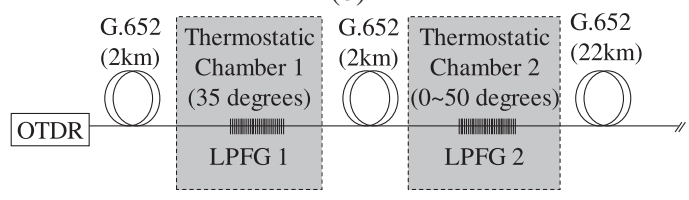

Fig. 1. Temperature measurement configurations: (a) single-point sensing, (b) multi-point sensing.

with temperature because of the thermal expansion and thermal elasticity of the tube [11].

When the transmission line is composed of the same type of fiber before and after the LPFG as shown in Fig. 1, the backscattered powers $P\left(z_{b}\right)$ and $P\left(z_{a}\right)(\mathrm{dB})$ before and after the LPFG can be expressed by the following equations:

$$
\begin{aligned}
& P\left(z_{b}\right) \cong 10 \log _{10}\left(\frac{P_{0} \alpha_{s} B W v_{g}}{2}\right)+10 \log _{10} \exp \left[-2 \int_{0}^{z_{b}} \alpha d z\right] \\
& P\left(z_{a}\right) \cong 10 \log _{10}\left(\frac{P_{0} \alpha_{s} B W v_{g}}{2}\right)+10 \log _{10} \exp \left[-2 \int_{0}^{z_{b}} \alpha d z\right]-20 \log _{10}\left(P_{L}(T)\right)
\end{aligned}
$$

Here, $P_{0}, \alpha_{s}$, and $B$ denote the input power, the local scattering coefficient, and the backscattered capture fraction, respectively. $W$ and $v_{g}$ are the pulse width of the OTDR and group velocity, respectively. $P_{L}(T)$ is the transmission loss of the LPFG at temperature $T$. The temperature-dependent insertion loss of the LPFG can then be measured from the difference in backscattered power before and after the LPFG [11].

Multi-point temperature sensing using LPFGs can be realized by cascading the LPFGs with transmission fibers. The backscattering from the transmission fiber after the most distant LPFG is then detected to measure the insertion loss of the LPFG. This means that the dynamic range $D$ of the OTDR must be greater than the total loss in the LPFGs and the transmission fibers. The length of the transmission fibers before and after the LPFGs must be longer than the distance resolution $L_{s}$ of the OTDR to allow the insertion loss to be measured. The distance resolution $L_{s}$ corresponds to the pulse width of the OTDR. Thus, when transmission fibers with the distance resolution $L_{s}$ of the OTDR are placed before and after the LPFGs, the dynamic range $D$ of the OTDR, the number of sensing points $N$, and the maximum loss $P_{\text {Lmax }}$ of the LPFG are related in the following manner:

$$
D \geq 10(N+1) \log _{10} \exp \left[\int_{0}^{L_{s}} \alpha d z\right]+10 N \log _{10}\left(P_{L \max }\right)+M .
$$

where $M(\mathrm{~dB})$ is the margin required to estimate the change in optical loss of the LPFG. Here, it is assumed that all LPFGs have the same length and the same temperature dependent loss, and that the maximum loss of the LFPGs is $P_{\text {Lmax }}$. 
The optical loss of the LPFG fabricated from a heat-shrinkable tube and a metric screw comprises losses caused by periodic refractive index modulation and by random microbending [11]. The microbending loss is negligible compared with the loss from periodic refractive index modulation [11]. Thus, the optical loss $P_{L}$ at the resonance wavelength of the LPFG can be expressed as follows [12]:

$$
P_{L} \cong \sin ^{2}(\kappa l) \text {. }
$$

where $\kappa$ and $l$ are the coupling coefficient and the length of the LPFG, respectively.

The design of the LPFG sensor was based on Eqs. (3) and (4).

Next, we describe how the number of sensors and the distance range for multipoint sensing were determined. When the pulse width of the OTDR was set to $1 \mu \mathrm{s}, L_{s}$ was found to be $0.1 \mathrm{~km}$. We assumed the maximum loss $P_{L m a x}$ of the LPFG, the dynamic range of the OTDR, the optical loss of the fiber, and the margin $M$ to be $1.4 \mathrm{~dB}, 32 \mathrm{~dB}, 0.2 \mathrm{~dB} / \mathrm{km}$, and $15 \mathrm{~dB}$, respectively. From Eq. (3), the maximum number of sensing points $N$ was found to be 11 . The sensing distance range was, therefore, $2.2 \mathrm{~km}(0.2 \times 11)$.

\section{Experimental results}

Fig. 1(a) shows the experimental setup used to measure the insertion loss of the LPFG at the temperature $T$. We used a commercially available metric screw (M3) with a pitch $\Lambda$ of $500 \mu \mathrm{m}$, a length of $5 \mathrm{~cm}$, and a diameter of $3 \mathrm{~mm}$. An LPFG was spliced between two optical fiber bobbins with a fiber length of $2 \mathrm{~km}$ (ITU-T G.652 fibers), and placed in a thermostatic chamber. The temperature was then changed from 0 to $50{ }^{\circ} \mathrm{C}$. An OTDR (Agilent E6003C) was used to derive the LPFG insertion loss $10 \log _{10}\left(P_{L}\right)(\mathrm{dB})$ from the backscattered power distribution. The OTDR wavelength was set at $1,555 \mathrm{~nm}$ while taking the resonance wavelength of the LPFG into account. The averaging time was $10 \mathrm{~s}$ and the pulse width was $300 \mathrm{~ns}$, corresponding to a spatial resolution of $30 \mathrm{~m}$.

Fig. 2(a) shows the temperature dependence of the backscattered power distributions of the fiber link containing the LPFG. It can be seen that the backscattered power after the LPFG changed as the temperature in the thermostatic chamber changed. The backscattered power variation before and after the LPFG, corresponding to the insertion loss, was dependent on the temperature. This is because the resonance loss is also temperature dependent [11].

Fig. 3(a) shows the relationship between the natural logarithm of the insertion loss $\ln \left(10 \log _{10}\left(P_{L}\right)\right)$ of the LPFG and the temperature $T$. The insertion loss of the LPFG fabricated from a heat-shrinkable tube showed a good fit with the exponential temperature function [11]. We, therefore, calculated the natural logarithm of the insertion loss. The open and filled circles show the insertion loss of the LPFG in the heating and cooling cycles, respectively. Measurements were taken three times in each cycle. The solid line was linearly fitted to the experimental data, measured in the heating cycle. It can be seen that the natural logarithm of the LPFG insertion loss decreased in direct proportion to the temperature. The experimental temperature dependence of the insertion loss $P_{L}$ can be expressed as follows:

$$
\ln \left(10 \log _{10}\left(P_{L}\right)\right)=-0.0452 T+0.160
$$



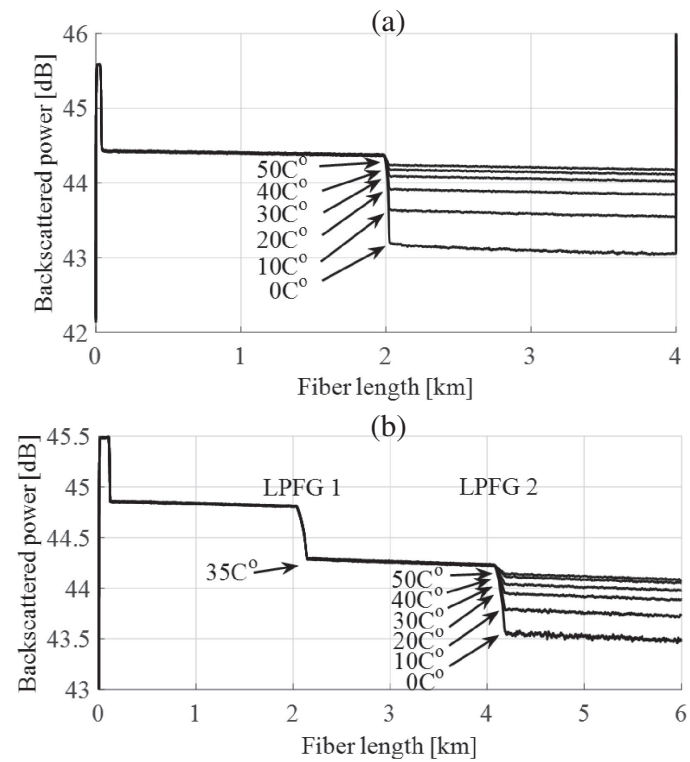

Fig. 2. Backscattered power distribution of the fiber link: (a) singlepoint sensing, (b) multi-point sensing.

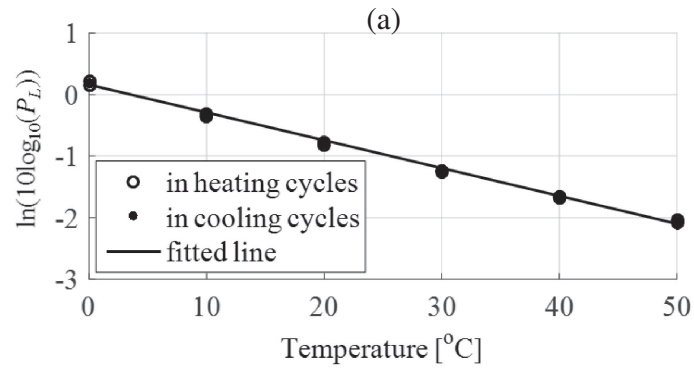

(b)

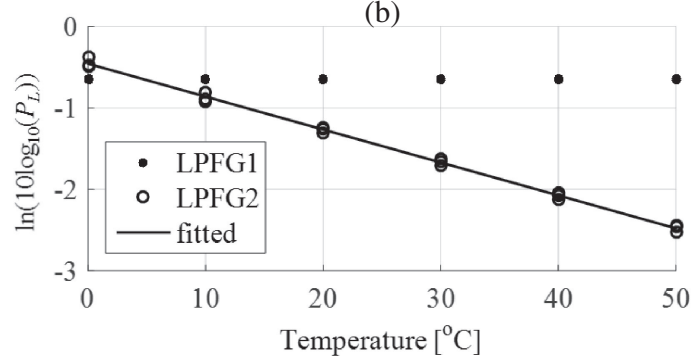

Fig. 3. Natural logarithm of insertion losses of LPFGs: (a) single-point sensing, (b) multi-point sensing.

This allows the temperature $T$ to be estimated from the natural logarithm of the insertion loss. The temperature in the thermostatic chamber was estimated from the insertion loss using Eq. (5), as shown in Fig. 2(a).

The temperature $T$ was obtained as follows:

$$
T=\left(0.160-\ln \left(10 \log _{10}\left(P_{L}\right)\right)\right) / 0.0452
$$

We defined the estimated temperature difference as the difference between the temperature in the thermostatic chamber and the estimated temperature obtained by substituting the measured insertion loss into Eq. (6). Across six measurements, the difference between the measured and estimated temperature was within $2{ }^{\circ} \mathrm{C}$. 
To investigate multi-point sensing, the temperatures of a fiber link comprising two LPFGs (LPFG1 and LPFG2) were measured, as shown in Fig. 1(b). The lengths of LPFG1 and LPFG2 were $5.3 \mathrm{~cm}$ and $4.0 \mathrm{~cm}$, respectively. Both were placed in thermostatic chambers. LPFG1 was kept at a constant $35^{\circ} \mathrm{C}$, while LPFG2 was heated from 0 to $50^{\circ} \mathrm{C}$. The backscattered power distribution was measured at the different temperatures of LPFG2 using the OTDR at a pulse width of $1 \mu \mathrm{s}$ and an averaging time of $10 \mathrm{~s}$.

Fig. 2(b) shows the backscattered power distributions of the fiber link containing the two LPFGs. The backscattered power difference before and after LPFG1 was almost unchanged because the temperature was kept constant. By contrast, the backscattered power difference before and after LPFG2 decreased as the temperature increased.

Fig. 3(b) shows the natural logarithm of the insertion losses of LPFG1 and LPFG2. Dots represent the natural logarithm of the insertion loss of LPFG1, at constant temperature, and the open circles represent that of LPFG2, at temperatures ranging from 0 to $50^{\circ} \mathrm{C}$. It can be seen that the insertion loss of LPFG1 was almost unchanged, whereas the insertion loss of LPFG2 changed in line with the temperature. This confirmed that the maximum loss of the LPFG could be varied by changing the length of the LPFG. The difference between the measured temperature in thermostatic chamber 2 and the estimated temperature was within $2^{\circ} \mathrm{C}$, and the experimental results showed good repeatability. This confirmed that the estimation of temperature distributions was possible using our proposed method.

\section{Conclusions}

We have proposed a simple and cost-effective temperature sensing based on a mechanical LPFG fabricated from a heat-shrinkable tube and a metric screw, both of which are commercially available. We have confirmed that the insertion loss of the LPFG measured using a conventional OTDR is temperature dependent. Our proposed technique has been used to measure the temperature of a thermostatic chamber with an accuracy of $2^{\circ} \mathrm{C}$. Multi-point temperature sensing has then been investigated, using two LPFGs. As in the single-point temperature measurement experiment, the accuracy has been found to be within $2{ }^{\circ} \mathrm{C}$. The experimental results have confirmed the potential use of LPFGs for temperature sensing.

\section{Acknowledgments}

The authors would like to thank Dr. I. Yamashita at the Kansai Electric Power Co., Inc. for a fruitful discussion and providing us with the thermostatic chamber. This work was supported by JSPS KAKENHI Grant Number 16K06307. 